\title{
The long noncoding RNA cancer susceptibility candidate 2 inhibits tumor progression in osteosarcoma
}

\author{
LINGYUN LU ${ }^{1}$, ZHEHAO DAI ${ }^{2}$, QING LUO ${ }^{3}$ and GUOHUA LV ${ }^{2}$ \\ ${ }^{1}$ Department of Orthopedics, The Fifth Hospital of Xiamen, Xiamen, Fujian 361101; ${ }^{2}$ Department of Spine Surgery, \\ The Second Xiangya Hospital, Central South University, Changsha, Hunan 410011; ${ }^{3}$ Molecular Oncology Laboratory, \\ Cancer Hospital, Affiliated Hospital of Zunyi Medical College, Zunyi, Guiyang 563000, P.R. China
}

Received October 24, 2016; Accepted June 15, 2017

DOI: $10.3892 / \mathrm{mmr} .2017 .8080$

\begin{abstract}
Long noncoding RNA (lncRNA) has been identified to serve a critical role in the development of various types of cancer. Cancer susceptibility candidate 2 (CASC2) is a cancer-associated lncRNA. However, whether CASC2 regulates osteosarcoma progression remains unclear. Reverse transcription-quantitative polymerase chain reaction, western blot, invasion and migration assays were used to evaluate the role of CASC2 in osteosarcoma. The present study reported that CASC2 may inhibit osteosarcoma development. Osteosarcoma tissues demonstrated reduced CASC2 expression compared with normal adjacent tissues. In addition, CASC2 transduction may decrease proliferation, migration and invasion of osteosarcoma cell lines whereas knockdown of CASC2 displayed opposing effects. Patients with low CASC2 levels were predicted to have a poor survival. In vivo implantation studies using pcDNA-CASC2 or short interfering-CASC2 exhibited decreased or increased tumor weight, respectively. These results suggested that CASC2 may serve as a potential tumor suppressor lncRNA in osteosarcoma and may provide potential insight into targeted intervention.
\end{abstract}

\section{Introduction}

Osteosarcoma (OS) may be fatal, particularly in adolescents (1). Greater than $50 \%$ of bone cancers are OS (2). Despite advances in chemotherapy and radiotherapy, the 5-year survival rate for OS patients remains low (3). The long-term prognosis of OS patients is poor, largely due to frequent metastasis, high recurrence rate and lack of effective therapeutic intervention (4). Therefore, a more detailed understanding of the underlying

Correspondence to: Dr Zhehao Dai, Department of Spine Surgery, The Second Xiangya Hospital, Central South University, 139 Middle Ren-min Road, Changsha, Hunan 410011, P.R. China

E-mail: daizh_csu@163.com

Key words: cancer susceptibility candidate 2, osteosarcoma, long noncoding RNA, malignancy mechanisms of OS development and effective therapeutics are required.

Long non-coding RNAs (lncRNAs) denote a class of RNAs longer than 200 nucleotides in length $(5,6)$, and may serve a role in biological processes including development and angiogenesis, differentiation and cell growth (7-9). Recent studies have suggested that lncRNAs may act as either oncogenes or tumor suppressors and contribute to the tumorigenesis of multiple cancers including OS $(10,11)$. For example, the lnc-RNA, metastasis associated lung adenocarcinoma transcript 1 , promotes the development of OS (12). Overexpression of the lncRNA MFI2 also increases the proliferation and progression of OS cells (11). Another report suggested an oncogenic role of HULC which is another lncRNA and is highly up-regulated in liver cancer in OS, when deregulated levels of it predicted poor prognosis (13). A well-characterized lncRNA, HOXA transcript at the distal tip, has also been demonstrated to be upregulated in OS cells and in patient samples and therefore contributes to the development of OS (14). Another lncRNA, tumor suppressor candidate 7, may instead inhibit OS progression via an unknown mechanism (15). Consistently, the lncRNA, long intergenic non-protein coding RNA 161, sensitizes OS cells to cisplatin-induced cell death and may be a candidate tumor suppressor (16). Therefore, lncRNAs may be applied for OS diagnosis and may act as novel therapeutic targets.

LncRNA, cancer susceptibility candidate 2 (CASC2) is located at chromosome 10q26, and is a novel cancer-associated lncRNA. Previously, decreased CASC2 expression and its tumor suppressive function has been demonstrated in various tumor types (17-21). However, the expression and potential role of CASC2 in OS remains unclear.

In the current study, the function of CASC 2 in OS was investigated. CASC2 expression was significantly decreased in OS tissues compared with normal adjacent tissues. The association of CASC2 levels with different clinicopathological features was also studied. Patients with greater CASC2 expression displayed improved survival. Increased CASC 2 expression impeded the proliferation, invasion and migration of OS cells. Consistently, increasing CASC2 expression also promoted apoptosis, whereas CASC2 knockdown exerted the opposite effect. In vivo implantation studies suggested that CASC2 overexpression decreased the oncogenic potential of OS cells. The data collectively 
suggested a tumor suppressive role of CASC 2 and may provide an insight into the diagnosis of OS.

\section{Materials and methods}

Cell culture and human samples. OS cell lines used in the current study, 143B, U-2 OS, KHOS-240S, D22, Saos-2, HOS and MG-63, and a normal cell line, hFOB, were purchased from the American Type Culture Collection (Manassas, VA, USA). The OS cells were maintained in RPMI-1640 medium (Tiangen Biotech Co., Ltd., Beijing, China) supplemented with 5\% fetal calf serum (FCS; Tiangen Biotech Co., Ltd.), streptomycin (50 $\mu \mathrm{g} / \mathrm{ml}$; Tiangen Biotech Co., Ltd.) and penicillin (200 U/ml, Sigma-Aldrich; Merck KGaA, Darmstadt, Germany) at $20^{\circ} \mathrm{C}$ and $5 \% \mathrm{CO}_{2}$. Matched fresh OS specimens and normal adjacent tissues were collected from 97 patients who had undergone resection at the Second Xiangya Hospital, Central South University (Hunan, China) between March 2010 and October 2014. Immediately after surgical resection, these tissues were stored at $-80^{\circ} \mathrm{C}$ until usage. None of the patients had received preoperative chemotherapy or radiotherapy. All patients signed formal consent forms. Overall survival was calculated from the day of primary surgery to death or last follow-up. The research performed using human specimens was reviewed and approved by the Ethics Committee of the Second Xiangya Hospital, Central South University (Hunan, China).

CASC2 overexpression and knockdown. A sequence of genomic DNA encoding the cDNA for CASC2 and flanking sequences was amplified by PCR and cloned into the EcoRI and XhoI (both purchased from Roche Diagnostics, Shanghai, China) sites of pCDNA3.1 vector (50 nM, Tiangen Biotech Co., Ltd.). For transfection, the empty pcDNA3.1 vector was used as a control. The sequence for CASC2 insert was: 5'-ACAACAAGAAACTTCCCCAAGGTATCA TTATAGTCTTTAGACTTCAGACACACACCACACCTC AAATATATACACAACTGAAAGGAAAATTAAGGAAGT TTTTCAAAGAACCCTATTCCGAGTAAGAAGTGTGTT GCATGAATTTCTAAGAGCCAGAAAATGCATGACACA GGAGAAGATGTACCCTCATCTGTTCAGTGAGAGATG TGCAAATCAACATCAACACAGAACTGCTGAAGAAAA AAAATATGTCTCTGAAAAGCAACTTATTCACTGGAG ATGTGAGGAGCCATCCGCACATCACAATTCTATAGA CATCAAACGCATGAAGCATTTCGGATCTGCTTTAAG ACTGAGGCAGACTTTCCATCTGGACACAGCCGACCA TCCATGTGTCATTACAATGAATCCAGCACTTCC-3'. The negative control siRNA was also introduced (Shanghai GenePharma, Co., Ltd., Shanghai, China). CASC2 small interfering RNAs (si-CASC2, $50 \mathrm{nM}$ ) were synthesized by Shanghai GenePharma, Co., Ltd. The si-CASC2 sequence was: 5'-AAGGCTGTATGCTGTATCATACCCTGTTCT CCCGGGTTTTGGCCACTGACTGACCCGGGAGAAG-3'.

Transfections were performed using Lipofectamine 2000 (Invitrogen; Thermo Fisher Scientific, Inc., Waltham, MA, USA) according to the manufacturer's protocol for $24 \mathrm{~h}$ at $20^{\circ} \mathrm{C}$.

Reverse transcription-quantitative polymerase chain reaction $(R T-q P C R)$. Total RNAs were isolated from 143B, U-2 OS, KHOS-240S, D22, Saos-2, HOS, MG-63 and hFOB cell lines and human samples with TRIzol reagent (Invitrogen; Thermo Fisher Scientific, Inc.). A total of $5 \mathrm{ng}$ RNA in a final volume of $10 \mu \mathrm{l}$ containing $5 \mathrm{mM}$ dNTP mix (Tiangen Biotech Co., Ltd.) was used to generate complementary DNA using the SYBR Premix Taq ${ }^{\mathrm{TM}}$ toolkit (Takara Biotechnology Co., Ltd., Dalian, China). The mixture was maintained at $70^{\circ} \mathrm{C}$ for $5 \mathrm{~min}$ and then a mixture composed of $5 \times \mathrm{RT}$ buffer, $50 \mathrm{U} / \mu 1$ reverse transcriptase (M-MLV), $100 \mathrm{U} / \mu 1 \mathrm{RNase}$ inhibitor was added (Tiangen Biotech Co., Ltd.). GAPDH was used as the control. Reactions were performed using the ABI PRISM ${ }^{\circledR} 7000$ Sequence Detection system (Applied Biosystems; Thermo Fisher Scientific, Inc.), according to the manufacturer's protocol. The expression of CASC2 was calculated using the $2^{-\Delta \Delta \mathrm{Cq}}$ method (22). The experiments were performed $\geq 3$ times. The primer sequences were as follows: Sense, 5'-GAATGC TAGCTTACG-3' and anti-sense, 5'-CTGAGTGCTTGACAT GT-3' for CASC2; sense, 5'-GATTAGCTCTGCACGTT-3' and anti-sense, 5'-ATGAGCATTACAGTGTT-3' for GAPDH. The cycling conditions were: $55^{\circ} \mathrm{C}$ for $2 \mathrm{~min}, 95^{\circ} \mathrm{C}$ for $15 \mathrm{~min}$ followed by 32 cycles of $95^{\circ} \mathrm{C}$ for $15 \mathrm{sec}$ and $60^{\circ} \mathrm{C}$ for $1 \mathrm{~min}$. Experiments were performed in triplicates.

Proliferation assay. In the proliferation study, a Cell Counting kit-8 (Dojindo Molecular Technologies, Inc., Kumamoto, Japan) was used. After treatment for 24 h, MG-63 and HOS cells were re-suspended and seeded into a 6-well plate $\left(1 \times 10^{5}\right.$ cells/well) for 5 days. A total of $20 \mu \mathrm{l} \mathrm{CCK-8} \mathrm{solution}$ was added into the culture to a final concentration of $10 \mathrm{mg} / \mathrm{ml}$ at $20^{\circ} \mathrm{C}$. The crystalline formazan product was resolved in $100 \mu 110 \% \mathrm{SDS}$ solution for one day at $20^{\circ} \mathrm{C}$. The optical density was detected at a wavelength of $490 \mathrm{~nm}$ and each assay was repeated three times.

Transwell invasion and migration assays. Cell invasion and migration assays were performed using 24-well Transwell chambers ( $8 \mu \mathrm{m}$ pore size; BD Biosciences, Franklin Lakes, NJ, USA). For the migration assay, $1 \times 10^{6}$ MG-63 and HOS cells were suspended in $100 \mu \mathrm{l}$ RPMI-1640 serum-free medium (Tiangen Biotech Co., Ltd., Beijing, China) and placed in the top chambers. Dulbecco's modified Eagle's medium (500 $\mu \mathrm{l}$, Sigma-Aldrich; Merck KGaA, Drmstadt, Germany) containing $10 \%$ FCS was added to the bottom chambers. Following $24 \mathrm{~h}$ of incubation at $37^{\circ} \mathrm{C}$, the cells that did not migrate into the pores were removed using a cotton swab, and cells on the lower surfaces of the membrane were stained with crystal violet $(0.40 \%)$ for $1 \mathrm{~h}$ at $20^{\circ} \mathrm{C}$ and evaluated. The invasion assay was similar to that of the migration assay except that the cell culture surface was firstly coated with Matrigel (BD Biosciences, Franklin Lakes, NJ, CA, USA). A fluorescence microscope (DM-IRB; Leica Microsystems GmbH, Wetzlar, Germany) was used to visualize the results and CELLCOUNTER (https://bitbucket.org/linora/cellcounter/downloads) was used for quantification. A total of five fields were assessed and experiments were performed in triplicates.

Cell cycle and apoptosis analysis. After a $48 \mathrm{~h}$ transfection, MG-63 and HOS cells were harvested and washed with cold PBS. Subsequently, cells were fixed with $70 \%$ ethanol at $4^{\circ} \mathrm{C}$ overnight. The fixed cells were then stained with propidium iodide (PI; Sigma-Aldrich; Merck KGaA) at $4^{\circ} \mathrm{C}$ for $30 \mathrm{~min}$ 
in the dark. The fraction of cells in $\mathrm{G}_{0} / \mathrm{G}_{1}, \mathrm{~S}$ and $\mathrm{G}_{2} / \mathrm{M}$ phases were measured using a fluorescence-activated cell sorting (FACS) instrument (BD Biosciences). The experiments were performed in triplicate.

For the apoptosis assay, transfected cells were examined using a fluorescein isothiocyanate-labeled Annexin V/PI Apoptosis Detection kit (Beyotime Institute of Biotechnology, Haimen, China) following the manufacturer's protocol. Flow cytometric analysis was performed immediately after staining. Data acquisition and analysis were performed using a FACS instrument (BD Biosciences).

In vivo implantation and immunohistochemistry. Transduction of CASC2 into $~ 5 \times 10^{6}$ MG-63 cells was performed using pcDNA3.1 vector as described above. The system was initially maintained for $24 \mathrm{~h}$ at $20^{\circ} \mathrm{C}$. Following this, cells were re-suspended and $1 \times 10^{6}$ cells were injected subcutaneously into the rear flank of nude mice ( $n=32$, female, $4 \sim 5$ weeks old, average weight $16.7 \mathrm{~g}$ ). Mice were housed at $20^{\circ} \mathrm{C}$, with $55-60 \%$ humidity and a light-dark cycle of 12/12 h. Ad libitum access to food and water was supplied. After 30 days, mice were sacrificed by sodium amobarbital (Sigma-Aldrich; Merck KGaA) overdose and solid tumors were resected and weighted. The tumor sections were examined by two experienced pathologists who were double blinded to the data. Nude mice were obtained from the Model Animal Research Center of Nanjing University (Nanjing, China). Tumor samples were fixed in $10 \%$ buffered formalin for $12 \mathrm{~h}$ at $20^{\circ} \mathrm{C}$, embedded in paraffin, and cut into $5 \mu \mathrm{m}$ sections. After deparaffinization and rehydration, antigens were retrieved with 1x Cytomation target retrieval solution (Dako; Agilent Technologies, Inc., Santa Clara, CA, USA) in a decloaker chamber at $95^{\circ} \mathrm{C}$ for $20 \mathrm{~min}$ and then at room temperature for $20 \mathrm{~min}$ followed by sequential rinsing with distilled water and PBS at room temperature. Slides were incubated with hydrogen peroxide for $5 \mathrm{~min}$ to quench endogenous hydrogen peroxidase activity. After rinsing twice with TBS with $0.1 \%$ Tween 20, slides were incubated with Ki-67 antibodies (1:1,000, cat. no. P6834, Sigma-Aldrich; Merck KGaA). Slides were counterstained with hematoxylin for $5 \mathrm{~min}$ and rinsed with distilled water. 3,3'-Diaminobenzidine (DAB) was used as a chromogen (cat. no. D8001, Sigma-Aldrich; Merck KGaA). Slides were evaluated under an optical light microscope (Olympus Corporation, Tokyo, Japan). Images were captured using x100 magnification. All the slides were manually scored as previously described (23). Briefly, three random sections from each animal of all groups were scored for the intensity of staining. $\mathrm{H}$-score were calculated as $\mathrm{H}$-score $=\Sigma(1+i) p i$, where $i$ is the intensity score ( 0 : no staining, 1 : weak staining, 2 : moderate staining; 3: Strong staining) and $p i$ is the percentage of cells exhibiting that intensity (24). The median value was used as the cutoff, as previously suggested (25).

Statistical analysis. Data are presented as the mean \pm standard deviation. Statistical significance was determined by Student's t-test or one-way analysis of variance followed by the least significant difference post hoc test, using SPSS software version 15.0 (SPSS, Inc., Chicago, IL, USA). P $<0.05$ was considered to indicate a statistically significant difference. The Kaplan-Meier survival curve was tested using log-rank test.
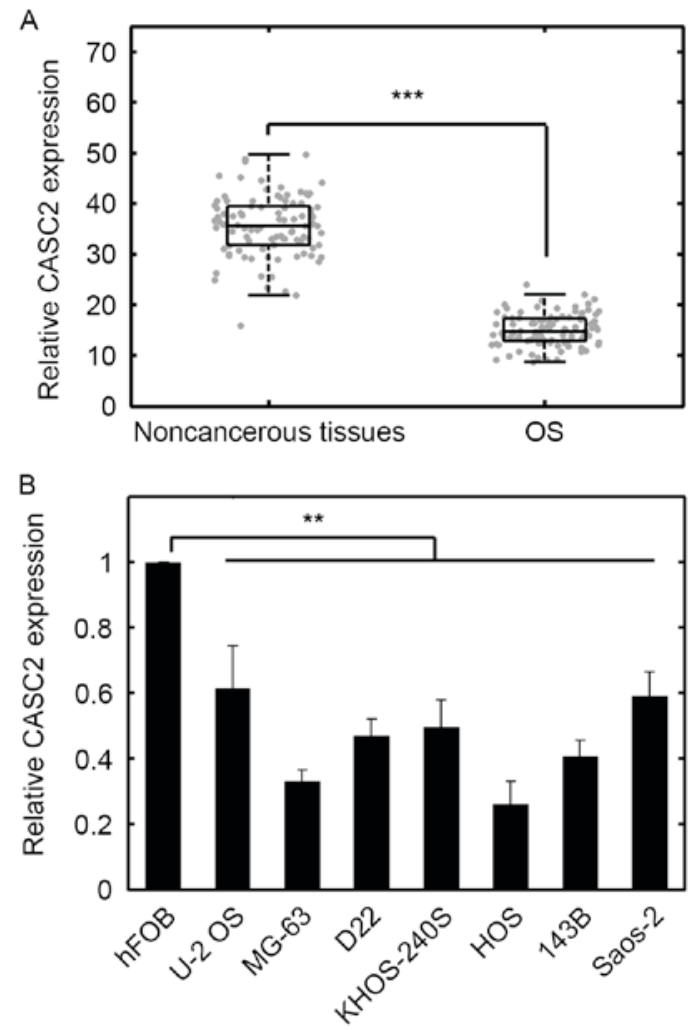

Figure 1. mRNA expression levels of the 1ncRNA CASC2 was downregulated in OS cells and specimens. (A) Reverse transcription-quantitative polymerase chain reaction results for 97 OS tissues and corresponding normal adjacent tissues. (B) Expression of CASC2 in the hFOB normal bone cell line and 7 OS cell lines, including 143B, U-2 OS, KHOS-240S, D22, Saos-2, HOS and MG-63. Data are presented as the mean \pm standard deviation. ${ }^{* * *} \mathrm{P}<0.01$ and ${ }^{* * *} \mathrm{P}<0.001$. OS, osteosarcoma; CASC2, cancer susceptibility candidate 2 gene.

Fisher exact test was used to evaluate the association between CASC2 and clinicopathological characteristics.

\section{Results}

IncRNA CASC2 is downregulated in specimens and OS cell lines. To quantify the expression levels of $C A S C 2$, RT-qPCR was conducted. The results indicated that the expression levels of $C A S C 2$ were significantly downregulated in OS samples compared with normal adjacent tissues (Fig. 1A; $\mathrm{P}<0.001$ ). In addition, in a number of OS cell lines, $C A S C 2$ was decreased compared with that of normal bone cells (Fig. 1B; $\mathrm{P}<0.01$ ). The median value was used as the cutoff, as previously suggested (25).

CASC2 protein expression in OS patient tissue was not significantly associated with age and gender of the patients (Table I); however, CASC2 levels were significantly associated with differentiation, tumor, nodes, metastasis (TNM) stages and metastasis (Table I). These results suggested that CASC2 expression was lower in OS cell lines and in human OS tissue samples, compared with normal controls. As downregulated CASC2 in OS tissues implies a tumor suppressive role for CASC2, the tumor cell lines with relatively low CASC2 expression may possibly exhibit greater tumorigenic potential compared with the cell lines with higher CASC2 expression levels. MG-63 and HOS cells exhibited the lowest CASC2 
A
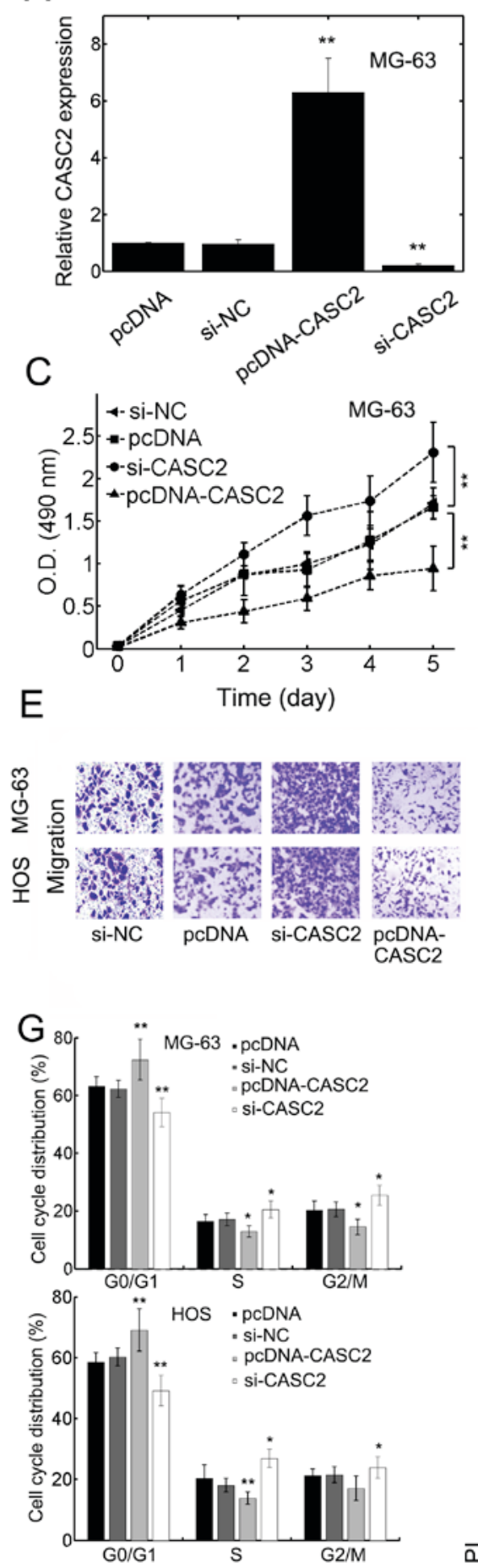

B
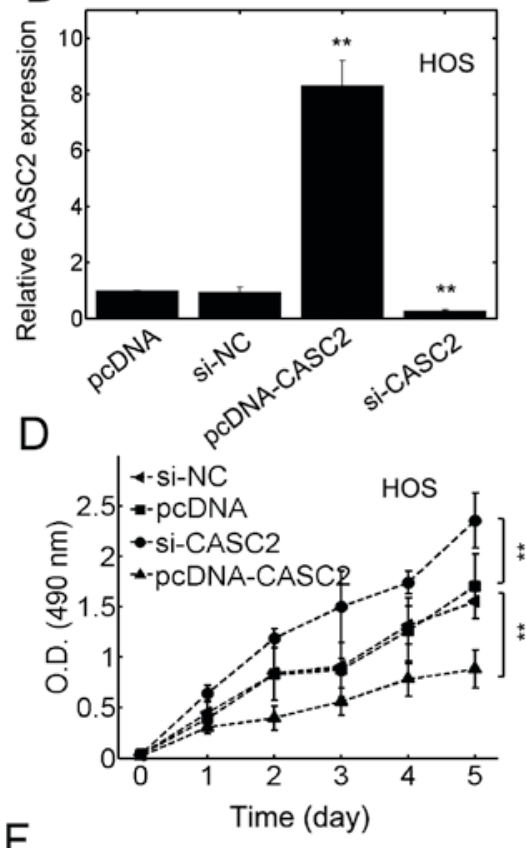

$\mathrm{F}$

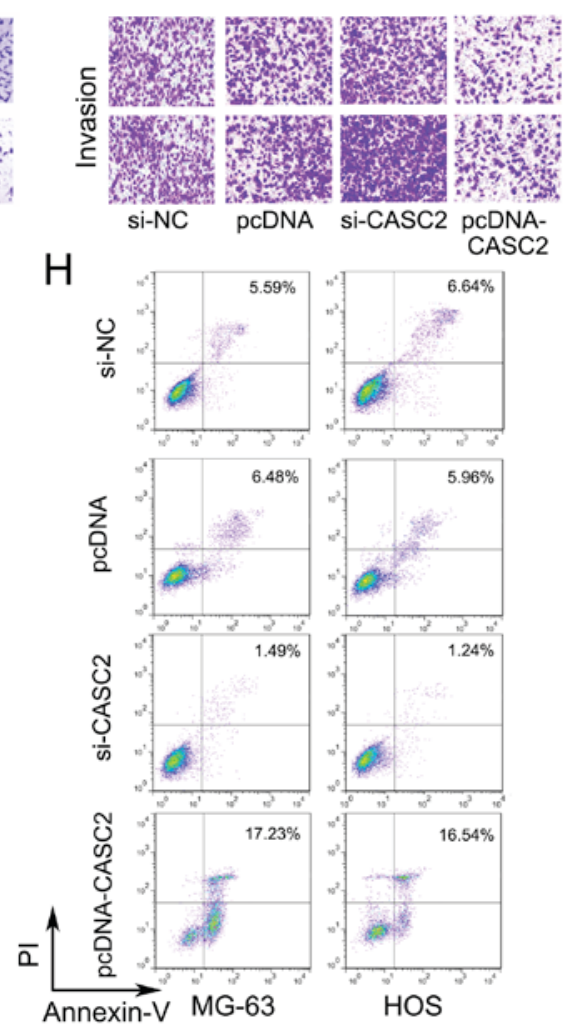

Figure 2. CASC2 overexpression inhibited osteosarcoma cell proliferation, migration and invasion. (A) MG-63 or (B) HOS cells were transfected with pcDNA, si-NC, pcDNA-CASC2 or si-CASC2. The mRNA expression levels of CASC2 was quantified using reverse transcription-quantitative polymerase chain reaction. A five-day proliferation assay for (C) MG-63 and (D) HOS cells transfected with pcDNA, si-NC, pcDNA-CASC2 or si-CASC2. (E) Transwell migration assays and (F) transwell invasion assays for MG-63 and HOS cells transfected with pcDNA, si-NC, pcDNA-CASC2 or si-CASC2. Representative images are displayed. (G) Cell cycle was measured for MG-63 (top graph) and HOS (bottom graph) cells transfected with pcDNA, si-NC, si-CASC2 or pcDNA-CASC2 by flow cytometry. (H) The percentage apoptotic cells were quantified using flow cytometry in MG-63 (left panel) and HOS (right panel) cells transfected with pcDNA, si-NC, si-CASC2 or pcDNA-CASC2. Data are presented as the mean \pm standard deviation. ${ }^{*} \mathrm{P}<0.05$ and ${ }^{* *} \mathrm{P}<0.01$. CASC2, cancer susceptibility candidate 2 gene; CASC2, cancer susceptibility candidate 2; si-CASC2, CASC2 short interfering RNA.

expression (Fig. 1B). To evaluate the potential tumor suppressive role of CASC2, we selected these two cell lines for further analysis.
CASC2 inhibits proliferation, migration and invasion of OS cells. It was then determined whether CASC2 affects the malignant phenotypes of OS cells. MG-63 and HOS cells 
Table I. Association between CASC2 expression and clinicopathological features.

CASC2 expression

\begin{tabular}{|c|c|c|c|c|}
\hline Clinicopathological features & No. & $\operatorname{High}[\mathrm{n}(\%)]$ & Low $[\mathrm{n}(\%)]$ & P-value \\
\hline \multicolumn{5}{|l|}{ Age } \\
\hline$<60$ & 47 & $26(55.3)$ & $21(44.7)$ & \multirow[t]{2}{*}{0.238} \\
\hline$\geq 60$ & 50 & $23(46.0)$ & $27(54.0)$ & \\
\hline \multicolumn{5}{|l|}{ Gender } \\
\hline Male & 74 & $36(48.6)$ & $38(51.4)$ & \multirow[t]{2}{*}{0.337} \\
\hline Female & 23 & $13(56.5)$ & $10(43.5)$ & \\
\hline \multicolumn{5}{|l|}{ Differentiation } \\
\hline Well/moderate & 60 & $38(63.3)$ & $22(36.7)$ & \multirow[t]{2}{*}{0.001} \\
\hline Poor & 37 & $11(29.7)$ & $26(70.3)$ & \\
\hline \multicolumn{5}{|l|}{ Metastasis } \\
\hline Absent & 23 & $8(24.2)$ & $25(75.8)$ & \multirow[t]{2}{*}{$<0.0001$} \\
\hline Present & 64 & $41(64.1)$ & $23(35.9)$ & \\
\hline \multicolumn{5}{|l|}{ TNM stage } \\
\hline $0 / \mathrm{I}$ & 45 & $30(66.7)$ & $15(33.3)$ & \multirow[t]{2}{*}{0.003} \\
\hline II/III/IV & 52 & $19(36.5)$ & $33(63.5)$ & \\
\hline
\end{tabular}

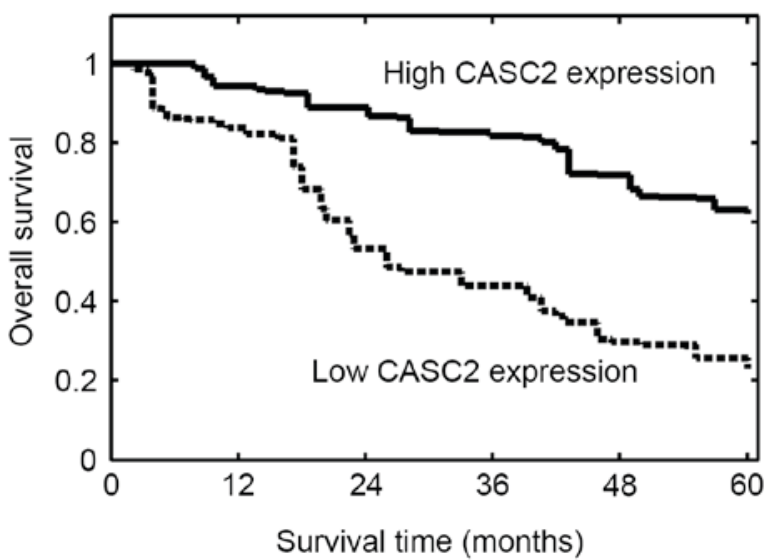

Figure 3. Kaplan-Meier survival curves for OS patients. OS patients with low CASC2 expression had a significantly shorter overall survival than those with high CASC2 expression levels $(\mathrm{P}<0.01$ using a log-rank test). OS, osteosarcoma; CASC2, cancer susceptibility candidate 2 .

were either untreated or transfected with pcDNA-CASC2 or si-CASC2. The mRNA expression levels of CASC2 in the cancer cells were then quantified. Transfection with pcDNA-CASC2 significantly upregulated the expression levels of $C A S C 2$ in MG-63 and HOS cells compared with the control (Fig. 2A and B). CASC2 overexpression significantly inhibited the proliferation of MG-63 and HOS cells compared with the empty vector control (Fig. $2 \mathrm{C}$ and $\mathrm{D} ; \mathrm{P}<0.01$ ). Knockdown of $C A S C 2$ using a specific siRNA significantly promoted the proliferation of MG-63 and HOS cells compared with the control (Fig. 2C and D). pcDNA-CASC2 transfection attenuated the migration of MG-63 and HOS cells, compared with the control (Fig. 2E). Knockdown of CASC2 led to elevated migration of MG-63 and HOS cells compared with the control

\section{A \\ B}
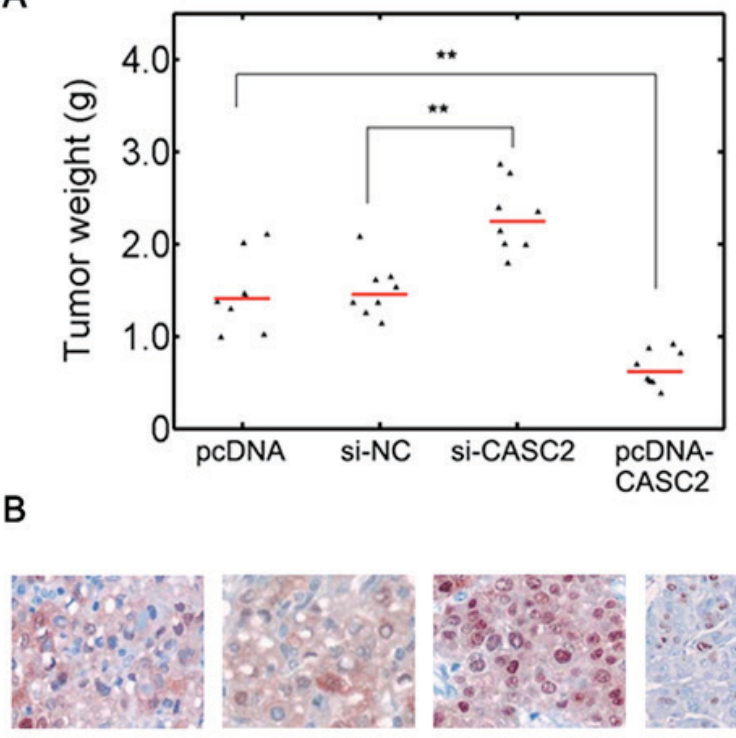

si-NC

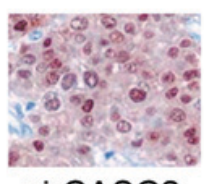

si-CASC2

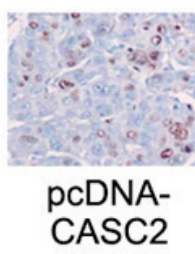

Figure 4. Overexpression of CASC2 may inhibit osteosarcoma growth in vivo. (A) Growth of solid tumors in nude mice injected with MG-63 cells transfected with pcDNA, si-NC, pcDNA-CASC2 or si-CASC2. Solid tumors were trimmed and weighed. Data are presented as the median tumor weight and $\mathrm{n}=8$ per group. ${ }^{* *} \mathrm{P}<0.01$. (B) Ki-67 immunostaining in MG-63 cells following in vivo implantation. MG-63 cells were transfected with pcDNA, si-NC, pcDNA-CASC2 or si-CASC2. CASC2, cancer susceptibility candidate 2 gene; si, short interfering.

(Fig. 2E). The effect of CASC2 on OS cell invasion was then investigated. The results demonstrated that CASC 2 knockdown resulted in increased invasion whereas overexpression of CASC2 inhibited invasion compared with the control (Fig. 2F). 
In addition, overexpression of $C A S C 2$ significantly increased the percentage of cells in G0/G1 phase of the cell cycle in both MG-63 and HOS cells compared with the control group (Fig. 2G). CASC2 knockdown cells displayed the opposite effect (Fig. 2G). In addition, CASC2 overexpression increased apoptosis of MG-63 and HOS cells (Fig. 2H), whereas CASC2 knockdown led to a decrease in the percentage of apoptotic cells, compared with the control (Fig. 2H). These results suggested that CASC2 inhibits the malignancy of OS cells via the inhibition proliferation, migration, invasion and induction of apoptosis.

Association between CASC2 expression and survival of OS patients. The prognostic significance of CASC2 expression in OS was investigated. The follow-up data was complete for all patients. The overall survival was evaluated from the day of primary surgery to mortality or to the last follow-up study. Based on these data, Kaplan-Meier survival curves were plotted. The results demonstrated that OS patients with lower CASC 2 expression had a significantly reduced overall survival compared with those with higher CASC2 expression ( $\mathrm{P}=0.002$; Fig. 3). These results suggested low expression of CASC2 in OS may be associated with poor survival.

CASC2 inhibits OS progression in vivo. In addition, in vivo implantation studies were conducted in order to further verify the effect of CASC2. It was observed that compared with the empty vector control, CASC2 overexpression significantly decreased the tumor weight (Fig. 4A; $\mathrm{P}<0.01$ ). CASC2 knockdown resulted in increased tumor weight compared with the control (Fig. 4A). Immunostaining for Ki-67 also identified that si-CASC2 treatment led to elevated proliferation in resected mice tumors whereas $C A S C 2$ overexpression reduced the proliferation (Fig. 4B). These results suggested that lncRNA CASC2 may serve a tumor suppressive role in vivo.

\section{Discussion}

Advances in biological technology have improved understanding of lncRNAs. A large number of lncRNAs have been identified to serve important roles in different malignant tumor types. Despite progress in cancer-associated research, the exact underlying molecular mechanisms of OS occurrence and development remain poorly understood.

Numerous efforts have been made to establishing an association between IncRNA expression and tumor development $(11,26-28)$. Owing to the complexity of the tumor microenvironment, lncRNA may serve diverse roles in carcinogenesis based on tumor type. Furthermore, as IncRNAs may also function as diagnostic or prognostic markers, even at the early stages of the tumorigenesis, determining the potential association between lncRNAs and various tumors is a challenge $(10,12-14)$. In the present study, the CASC2 expression in OS tissues was investigated, and results indicated that $C A S C 2$ was substantially downregulated in cancerous tissues compared with normal adjacent ones. $C A S C 2$ overexpression decreased the malignant potential of OS MG-63 and HOS cells. In addition, siRNA-mediated $C A S C 2$ knockdown increased the oncogenic properties of OS cells, including proliferation, migration and invasion.
Higher CASC2 expression was associated with improved prognosis of OS patients. The effect of CASC2 in an in vivo model was investigated, which suggested that $\mathrm{CASC} 2$ serves a tumor suppressive role.

The IncRNA CASC2 was originally identified as a candidate tumor suppressor transcript in endometrial cancer in 2004 (21). Wang et al (18) observed that CASC2 was markedly downregulated in human glioma cell lines. Overexpression of $C A S C 2$ inhibited the malignancy of glioma cells including proliferation and invasion (18). Low CASC2 expression levels may additionally serve as an unfavorable predictor for overall survival of patients with non-small cell lung cancer (NSCLC). Overexpression of CASC2 has been previously reported to inhibit NSCLC progression in vitro and in vivo (20). In colorectal cancer, decreased CASC2 expression was significantly associated with advanced TNM stage (19). As a result, CASC2 may behave as a potential tumor suppressive lncRNA in various types of cancer. In the present study, a role of lncRNA CASC 2 in OS was reported and this further emphasized its potential function in controlling cancer progression.

In conclusion, the function of IncRNA CASC2 in OS was investigated in the present study and results suggested that it may serve a tumor suppressive role. Low CASC2 expression was significantly associated with OS progression. Overexpression of CASC2 may attenuate the malignant phenotypes of OS in OS cell lines in addition to in tumor xenograft tissues. The data suggested that CASC2 may serve as a novel prognostic biomarker and a promising therapeutic target for further intervention. With more advance strategies such as high-throughput technology, more detailed mechanisms regarding how CASC2 inhibits tumor progression may be defined.

\section{Acknowledgements}

The present study was supported by the Science and Technology Plan of Xiamen (grant no. 3502Z20154067).

\section{References}

1. Heare T, Hensley MA and Dell'Orfano S: Bone tumors: Osteosarcoma and Ewing's sarcoma. Curr Opin Pediatr 21: 365-372, 2009.

2. Shackleton M, Quintana E, Fearon ER and Morrison SJ: Heterogeneity in cancer: Cancer stem cells versus clonal evolution. Cell 138: 822-829, 2009.

3. Levings PP, McGarry SV, Currie TP, Nickerson DM, McClellan S, Ghivizzani SC, Steindler DA and Gibbs CP: Expression of an exogenous human Oct-4 promoter identifies tumor-initiating cells in osteosarcoma. Cancer Res 69: 5648-5655, 2009.

4. Hattinger CM, Fanelli M, Tavanti E, Vella S, Ferrari S, Picci P and Serra M: Advances in emerging drugs for osteosarcoma. Expert Opin Emerg Drugs 20: 495-514, 2015.

5. Wang KC and Chang HY: Molecular mechanisms of long noncoding RNAs. Mol Cell 43: 904-914, 2011.

6. Ernst $\mathrm{C}$ and Morton CC: Identification and function of long non-coding RNA. Front Cell Neurosci 7: 168, 2013.

7. Fatica A and Bozzoni I: Long non-coding RNAs: New players in cell differentiation and development. Nat Rev Genet 15: 7-21, 2014.

8. Maass PG, Luft FC and Bähring S: Long non-coding RNA in health and disease. J Mol Med (Berl) 92: 337-346, 2014.

9. Carpenter S, Aiello D, Atianand MK, Ricci EP, Gandhi P, Hall LL, Byron M, Monks B, Henry-Bezy M, Lawrence JB, et al: A long noncoding RNA mediates both activation and repression of immune response genes. Science 341: 789-792, 2013. 
10. Zhou Q, Chen F, Fei Z, Zhao J, Liang Y, Pan W, Liu X and Zheng D: Genetic variants of IncRNA HOTAIR contribute to the risk of osteosarcoma. Oncotarget 7: 19928-19934, 2016.

11. Yin Z, Ding H, He E, Chen J and Li M: Overexpression of long non-coding RNA MFI2 promotes cell proliferation and suppresses apoptosis in human osteosarcoma. Oncol Rep 36: 2033-2040, 2016.

12. Luo W, He H, Xiao W, Liu Q, Deng Z, Lu Y, Wang Q, Zheng Q and Li Y: MALAT1 promotes osteosarcoma development by targeting TGFA via MIR376A. Oncotarget 7: 54733-54743, 2016.

13. Uzan VR, Lengert A, Boldrini É, Penna V, Scapulatempo-Neto C, Scrideli CA, Filho AP, Cavalcante CE, de Oliveira CZ, Lopes LF and Vidal DO: High expression of HULC is associated with poor prognosis in osteosarcoma patients. PLoS One 11: e0156774, 2016

14. Li F, Cao L, Hang D, Wang F and Wang Q: Long non-coding RNA HOTTIP is up-regulated and associated with poor prognosis in patients with osteosarcoma. Int J Clin Exp Pathol 8 11414-11420, 2015.

15. Cong M, Li J, Jing R and Li Z: Long non-coding RNA tumor suppressor candidate 7 functions as a tumor suppressor and inhibits proliferation in osteosarcoma. Tumour Biol 37: 9441-9450, 2016.

16. Wang Y, Zhang L, Zheng X, Zhong W, Tian X, Yin B, Tian K and Zhang W: Long non-coding RNA LINC00161 sensitises osteosarcoma cells to cisplatin-induced apoptosis by regulating the miR-645-IFIT2 axis. Cancer Lett 382: 137-146, 2016.

17. Cao Y, Xu R, Xu X, Zhou Y, Cui L and He X: Downregulation of IncRNA CASC2 by microRNA-21 increases the proliferation and migration of renal cell carcinoma cells. Mol Med Rep 14: 1019-1025, 2016.

18. Wang P, Liu YH, Yao YL, Li Z, Li ZQ, Ma J and Xue YX: Long non-coding RNA CASC2 suppresses malignancy in human gliomas by miR-21. Cell Signal 27: 275-282, 2015.

19. Huang $\mathrm{G}, \mathrm{Wu} \mathrm{X}, \mathrm{Li} \mathrm{S}, \mathrm{Xu} X, \mathrm{Zhu} \mathrm{H}$ and Chen X: The long noncoding RNA CASC2 functions as a competing endogenous RNA by sponging miR-18a in colorectal cancer. Sci Rep 6: 26524, 2016.
20. He X, Liu Z, Su J, Yang J, Yin D, Han L, De W and Guo R: Low expression of long noncoding RNA CASC2 indicates a poor prognosis and regulates cell proliferation in non-small cell lung cancer. Tumour Biol 37: 9503-9510, 2016.

21. Baldinu P, Cossu A, Manca A, Satta MP, Sini MC, Rozzo C, Dessole S, Cherchi P, Gianfrancesco F, Pintus A, et al: Identification of a novel candidate gene, CASC2, in a region of common allelic loss at chromosome 10q26 in human endometrial cancer. Hum Mutat 23: 318-326, 2004.

22. Livak KJ and Schmittgen TD: Analysis of relative gene expression data using real-time quantitative PCR and the 2(-Delta Delta C(T)) method. Methods 25: 402-408, 2001.

23. Godbole GB, Modi DN and Puri CP: Regulation of homeobox A10 expression in the primate endometrium by progesterone and embryonic stimuli. Reproduction 134: 513-523, 2007.

24. Cheon KW, Lee HS, Parhar IS and Kang IS: Expression of the second isoform of gonadotrophin-releasing hormone (GnRH-II) in human endometrium throughout the menstrual cycle. Mol Hum Reprod 7: 447-452, 2001.

25. Ling H, Spizzo R, Atlasi Y, Nicoloso M, Shimizu M, Redis RS, Nishida N, Gafà R, Song J, Guo Z, et al: CCAT2, a novel noncoding RNA mapping to 8q24, underlies metastatic progression and chromosomal instability in colon cancer. Genome Res 23: 1446-1461, 2013.

26. Fan H, Liu G, Zhao C, Li X and Yang X: Transcription factor Oct4 promotes osteosarcoma by regulating lncRNA AK055347. Oncol Lett 13: 396-402, 2017.

27. Li Z, Shen J, Chan MT and Wu WK: TUG1: A pivotal oncogenic long non-coding RNA of human cancers. Cell Prolif 49: 471-475, 2016.

28. Ye K, Wang S, Zhang H, Han H, Ma B and Nan W: Long noncoding RNA GAS5 suppresses cell growth and epithelial-mesenchymal transition in osteosarcoma by regulating the miR-221/ARHI pathway. J Cell Biochem: May 18, 2017 (Epub ahead of print). 